RI-3-96

September, 1996

\title{
A New Family of Solvable Self-Dual Lie Algebras
}

\author{
Oskar Pelc \\ Racah Institute of Physics, The Hebrew University \\ Jerusalem, 91904, Israel \\ E-mail: oskar@shum.cc.huji.ac.il
}

\begin{abstract}
A family of solvable self-dual Lie algebras is presented. There exist a few methods for the construction of non-reductive self-dual Lie algebras: an orthogonal direct product, a double-extension of an Abelian algebra, and a Wigner contraction. It is shown that the presented algebras cannot be obtained by these methods.
\end{abstract}




\section{Introduction}

A self-dual Lie algebra $\mathcal{A}$ is a finite-dimensional Lie algebra that admits an invariant metric, i.e., a symmetric non-degenerate bilinear form $(\cdot, \cdot)$ that is invariant under the adjoint action of the corresponding group:

$$
\left(g x_{1} g^{-1}, g x_{2} g^{-1}\right)=\left(x_{1}, x_{2}\right), \quad \forall x_{i} \in \mathcal{A},
$$

for any $g$ in the group, or equivalently,

$$
\left(\left[y, x_{1}\right], x_{2}\right)=-\left(x_{1},\left[y, x_{2}\right]\right), \quad \forall x_{i} \in \mathcal{A},
$$

for any $y \in \mathcal{A}$ (other common names for such algebras are "orthogonal" and "symmetricself-dual").

Self-dual Lie algebras are important in physics. In string theory, one needs a (highest weight) representation of the Virasoro algebra [1]. An important source of such representations is the affine Sugawara construction [2], starting from a Kac-Moody algebra [3]. Self-dual Lie algebras are precisely the Lie algebras that are needed for such a construction [ [4, 5]. From the point of view of two-dimensional conformal quantum field theories, a self-dual Lie algebra is the mathematical structure needed for the construction of Wess-Zumino-Novikov-Witten (WZNW) models [6].

The best known families of self-dual algebras are the semi-simple algebras [where the (essentially unique) invariant metric is the Killing form] and the Abelian algebras (for which every metric is trivially invariant). However, these are not the only ones. Any self-dual Lie algebra can be constructed, using semi-simple and Abelian algebras, by a sequence of construction steps, each of which is either an orthogonal direct sum (i.e., a direct sum equipped with the natural direct sum metric) or a procedure called double extension [7] (see also [5]). Self-dual algebras that are not orthogonal direct sums are called indecomposable and those that are also non-reductive and are not double extensions of an Abelian algebra will be called deeper (following [5]), since their construction involves more then one step of double-extension. Another method to obtain self-dual algebras is through a Wigner contraction [8], as described in [9]. A self dual algebra obtained by this method is always a double extension of an Abelian algebra and therefore is not a deeper algebra.

In this paper we present a new (as far as we know) infinite family of indecomposable, non-reductive (in fact, solvable), self-dual algebras $\left\{\mathcal{A}_{3 m}\right\}$. We show that these algebras (except the first two) are deeper algebras, in the sense defined above. Among the known self dual algebras, deeper algebras are rare (in fact, we do not know any other examples). The reason for this may be the absence of a practical method to construct such algebras (as will be explained in section IV). Therefore the family presented here may provide a valuable test-ground in the study of the properties and structure of general self-dual algebras and the physical models based on them [10].

The algebra

$$
\mathcal{A}_{n} \equiv \operatorname{sp}\left\{T_{i}\right\}_{0 \leq i \leq n}
$$


is defined by the Lie bracket

$$
\left[T_{i}, T_{j}\right]=\left\{\begin{array}{cl}
(\widehat{i-j}) T_{i+j} & i+j \leq n \\
0 & \text { otherwise }
\end{array}\right.
$$

where $\hat{i} \equiv i \bmod 3$ is chosen to be in $\{-1,0,1\}$. When $\hat{n}=0$, the metric

$$
\left(T_{i}, T_{j}\right)=\delta_{i+j-n}+b \delta_{i} \delta_{j}
$$

is an invariant metric on $\mathcal{A}_{n}$ (for arbitrary $b$ ).

In section II we define the algebras $\mathcal{A}_{n}$ and prove that (for $\hat{n}=0$ ) these are indeed self-dual Lie algebras. In section III we find all the ideals of $\mathcal{A}_{n}$. This result is used in section $\mathrm{V}$, where we check which of these algebras is a double extension of an Abelian algebra or a result of a Wigner contraction. Before that, in section IV, we describe these two methods briefly, emphasizing the gap that the algebras presented here may help to reduce. Finally, in section VI, we comment about possible generalizations.

\section{The Algebra $\mathcal{A}_{n}$}

Consider a vector space, equipped with the basis $\left\{T_{i}\right\}_{i \in \mathbb{Z}}$ and the following "Lie bracket":

$$
\left[T_{i}, T_{j}\right]=(\widehat{i-j}) T_{i+j}
$$

where $\hat{i} \equiv i \bmod 3$ is chosen to be in $\{-1,0,1\}$. The map $i \rightarrow \hat{i}$ is almost a ring homomorphism $\mathbb{Z} \rightarrow \mathbb{Z}$ : it preserves multiplication,

$$
\widehat{(i j)}=\hat{i} \hat{j}
$$

and almost preserves addition:

$$
\begin{gathered}
(\widehat{i+j})=(\widehat{i}+\hat{j}) \quad, \quad \widehat{(-i})=-\hat{i} \\
(\hat{i-j})=0 \Longleftrightarrow \hat{i}=\hat{j}
\end{gathered}
$$

(but note that $\hat{1}+\hat{1} \neq(\widehat{1+1})[1])$. These are the properties that will be used in the following. Particularly useful will be the property

$$
\hat{i}=\hat{j} \Longleftrightarrow(\hat{i+k})=(\widehat{j+k})
$$

which follows from (8).

The bracket is manifestly anti-symmetric so to obtain a Lie algebra, there remains to verify the Jacobi identity. Since

$$
\left[\left[T_{i}, T_{j}\right], T_{k}\right]=\hat{c}_{i j k} T_{i+j+k} \quad, \quad c_{i j k} \equiv(i-j)(i+j-k),
$$


the Jacobi identity takes the form

$$
\hat{c}_{i j k}+\hat{c}_{j k i}+\hat{c}_{k i j}=0
$$

This identity holds without the 'hats', therefore, by (7),

$$
\hat{c}_{i j k}+\hat{c}_{j k i}+\hat{c}_{k i j}=0 \bmod 3
$$

so (11) can be false only when

$$
\hat{c}_{i j k}=\hat{c}_{j k i}=\hat{c}_{k i j}= \pm 1 \text {. }
$$

$\hat{c}_{i j k}=1$ is equivalent to $(\widehat{i-j})=(i+\widehat{j}-k)= \pm 1$ and, therefore, also to

$$
i=j \pm 1 \bmod 3 \quad, \quad k=-j \bmod 3
$$

and this cannot hold simultaneously for all the tree cyclic permutations of $\{i j k\}$. Replacing $i \leftrightarrow j$, one obtains the same result for $\hat{c}_{i j k}=-1$. Therefore, the Jacobi identity holds and the above algebra is indeed a Lie algebra (over the integers).

Let us consider the subalgebra

$$
\mathcal{A}_{\infty} \equiv \operatorname{sp}\left\{T_{i}\right\}_{i \geq 0}
$$

Dividing by the ideal $\operatorname{sp}\left\{T_{i}\right\}_{i>n}$ (for some positive integer $n$ ), one obtains the finitedimensional Lie algebra

$$
\mathcal{A}_{n} \equiv \operatorname{sp}\left\{T_{i}\right\}_{0 \leq i \leq n}
$$

with the Lie bracket

$$
\left[T_{i}, T_{j}\right]=\left\{\begin{array}{cl}
(\widehat{i-j}) T_{i+j} & i+j \leq n, \\
0 & \text { otherwise. }
\end{array}\right.
$$

From now on we restrict our attention to such an algebra. It is a solvable algebra, $T_{0}$ being the only non-nilpotent generator and it possesses a $\mathbb{Z}$-grading: $\operatorname{deg}\left(T_{i}\right)=i$ (inherited from the original infinite-dimensional algebra).

We would like to find an invariant metric $(\cdot, \cdot)$ on $\mathcal{A}_{n}$. Using (3), the invariance condition

$$
\left(\left[T_{k}, T_{i}\right], T_{j}\right)+\left(T_{i},\left[T_{k}, T_{j}\right]\right)=0
$$

takes the form

$$
(\widehat{k-i})\left(T_{k+i}, T_{j}\right)+(\widehat{k-j})\left(T_{k+j}, T_{i}\right)=0
$$

(here $T_{i} \equiv 0$ for $i>0$ ) and, in particular, for $k=0$ :

$$
(\widehat{(-i)} \widehat{+}(\widehat{-j}))\left(T_{i}, T_{j}\right)=0
$$

which, by eqs. (7,8), is equivalent to

$$
(\widehat{i+j})\left(T_{i}, T_{j}\right)=0
$$


This means that two out of each three "reversed" (right-up-to-left-down) diagonals vanish. Let us look for a metric with only one non-vanishing diagonal. To obtain a non-degenerate form, this must be the central diagonal and according to (17), this is possible only for $\hat{n}=0$. We, therefore, concentrate on this case and consider a metric of the form

$$
\left(T_{i}, T_{j}\right)=\omega_{j} \delta_{i+j, n} \quad, \quad \omega_{n-j}=\omega_{j} \neq 0 .
$$

For such a metric the invariance condition (15) takes the form

$$
(\widehat{k-i}) \omega_{j}+(\widehat{k-j}) \omega_{i}=0, \quad \forall i+j+k=n
$$

and using $\hat{n}=0$, one obtains

$$
(2 \widehat{i+j}) \omega_{j}+(2 \widehat{j+i}) \omega_{i}=0
$$

First we take $\hat{j}=0$, which gives

$$
\hat{i}\left(\omega_{i}+\hat{2} \omega_{j}\right)=0
$$

and this implies (since $\hat{2} \neq 0$ )

$$
\omega_{i}=\left\{\begin{array}{cc}
\omega_{i}=-\hat{2} \omega_{0} & \hat{i} \neq 0 \\
\omega_{i}=\omega_{0} & \hat{i}=0 .
\end{array}\right.
$$

Using this result we take $\hat{i}, \hat{j} \neq 0$ in $(20)$ and obtain

$$
\hat{2} \cdot \hat{3}(\hat{i+j}) \omega_{0}=0
$$

which is satisfied, since $\hat{3}=0$. Also, since $-\hat{2}=1$, we have $\omega_{i}=\omega_{0}, \forall i$. To summarize, we proved the following.

\section{Lemma:}

$A$ (non-degenerate) invariant metric on $\mathcal{A}_{n}$ with only one (reversed) diagonal exists iff $\hat{n}=0$ and it is proportional to

$$
\left(T_{i}, T_{j}\right)=\delta_{i+j-n}
$$

Note that one can add to the metric a multiple of the Killing form, obtaining

$$
\left(T_{i}, T_{j}\right)=\delta_{i+j-n}+b \delta_{i} \delta_{j}
$$

(with $b$ arbitrary). The appearance of the second term can also be seen as a result of the (automorphic) change of basis

$$
T_{0} \rightarrow T_{0}+\frac{1}{2} b T_{n}
$$




\section{The Ideals in $\mathcal{A}_{n}$}

In this section we continue to analyze the algebra $\mathcal{A}_{n}$, looking for all its ideals and concluding that, for $\hat{n}=0$, the only ideals are of the form

$$
\mathcal{A}_{m, n} \equiv \operatorname{sp}\left\{T_{i}\right\}_{i=m}^{n}
$$

This will be important later, when we will check if these algebras are double extensions of Abelian algebras. The grading on $\mathcal{A}_{n}\left(\operatorname{deg}\left(T_{i}\right)=i\right)$ will play a central role in the following and will be called "charge". The adjoint action of $T_{i}$ increases the charge by $i$. Note that there are only positive charges, so that the adjoint action cannot decrease the charge. This proves that $\mathcal{A}_{m, n}$ (for any $m$ ) is indeed an ideal.

Let $\mathcal{J}$ be an ideal in $\mathcal{A}_{n}$. We choose a basis for $\mathcal{J}$ such that each element has a different minimal charge (this can be easily accomplished) and, therefore, can be labeled by it. We, therefore, have (after an appropriate normalization)

$$
\mathcal{J}=\operatorname{sp}\left\{S_{\alpha}\right\}, \quad S_{\alpha}-T_{\alpha} \in \mathcal{A}_{\alpha+1, n} .
$$

Isolating in $\mathcal{J}$ the maximal ideal of the form $\mathcal{A}_{m, n}$, we obtain

$$
\mathcal{J}=\operatorname{sp}\left\{S_{\alpha}\right\}_{\alpha \in \mathbf{A}} \bigoplus \mathcal{A}_{m, n} \quad, \quad m-1 \notin \mathbf{A} .
$$

Observe that this implies that for any element in $\mathcal{J}$ that is not in $\mathcal{A}_{m, n}$, its minimal charge is in $\mathbf{A}$.

The choice $\mathbf{A}=\emptyset$ (the empty set) corresponds to the "trivial" solution $\mathcal{J}=\mathcal{A}_{m, n}$. In the following we look for other solutions, i.e., with $\mathbf{A} \neq \emptyset$. This also implies $\max (\mathbf{A})<$ $m-1$. We are going to explore the restrictions on the $S_{\alpha}$ 's implied by the claim that $\mathcal{J}$ is an ideal in $\mathcal{A}_{n}$. Since $\mathcal{A}_{m, n}$ is an ideal by itself, the only restrictions come from

$$
\left[T_{i}, S_{\alpha}\right] \in \mathcal{J} \quad \forall \alpha \in \mathbf{A} \quad, \quad i=0, \ldots, n
$$

$\mathcal{J}$ contains all terms with charge of at least $m$, therefore, restrictions will arise only in terms in the commutator with smaller charge. For $i \geq m-\alpha$ there are no such terms. As the charge $i$ decreases, there will be more non-trivial terms, therefore, we will start from the higher charges.

For $i=m-\alpha-1$ we have (in the following, " $\simeq$ " means "equality up to an element of $\left.\mathcal{A}_{m, n} "\right)$ :

$$
\left[T_{m-\alpha-1}, S_{\alpha}\right] \simeq\left[T_{m-\alpha-1}, T_{\alpha}\right]=(m-\widehat{2 \alpha}-1) T_{m-1}
$$

(here and in other similar cases the hat should be applied to the whole expression between parentheses). $T_{m-1} \notin \mathcal{J}$ (otherwise $\mathcal{A}_{m-1, n} \subset \mathcal{J}$ ), therefore,

$$
(m-\widehat{2 \alpha}-1)=0 \text {. }
$$

Using eqs. (7.,8), this is equivalent to

$$
\hat{\alpha}=-\widehat{(2 \alpha)}=-(\widehat{m-1}),
$$


and since this is true for all $\alpha \in \mathbf{A}$, we also have

$$
\hat{\alpha}_{1}=\hat{\alpha}_{2}, \quad \forall \alpha_{1}, \alpha_{2} \in \mathbf{A} .
$$

Next, for $i=m-\alpha-2$ we have [using eqs. (31) and (7)]

$$
\left[T_{m-\alpha-2}, S_{\alpha}\right] \simeq\left[T_{m-\alpha-2}, T_{\alpha}+s_{\alpha}^{\alpha+1} T_{\alpha+1}\right]=-T_{m-2}+s_{\alpha}^{\alpha+1} T_{m-1}
$$

This implies that $m-2$ is a minimal charge of an element of $\mathcal{J}$, therefore, $m-2 \in \mathbf{A}$. Substituting $\alpha=m-2$ in (33), we obtain

$$
\left[T_{0}, S_{m-2}\right] \simeq-T_{m-2}+s_{m-2}^{m-1} T_{m-1} \simeq-S_{m-2}+2 s_{m-2}^{m-1} T_{m-1},
$$

and this implies $s_{m-2}^{m-1}=0$, so with no loss of generality, we can choose

$$
S_{m-2}=T_{m-2}
$$

Finally, for $i=m-\alpha-3$ and $m-2>\alpha \in \mathbf{A}$, we have

$$
\left[T_{m-\alpha-3}, S_{\alpha}\right] \simeq\left[T_{m-\alpha-3}, T_{\alpha}+s_{\alpha}^{\alpha+1} T_{\alpha+1}+s_{\alpha}^{\alpha+2} T_{\alpha+2}\right]=T_{m-3}+s_{\alpha}^{\alpha+2} T_{m-1},
$$

and as before this should imply that $m-3 \in \mathbf{A}$ (being the minimal charge of an element of $\mathcal{J}$ ). However, according to eq. (32), this is impossible since $m-2 \in \mathbf{A}$. Therefore, $\mathbf{A}$ contains no elements other then $m-2$ and $\mathcal{J}$ is of the form

$$
\mathcal{J}=\operatorname{sp}\left\{T_{m-2}\right\} \oplus \mathcal{A}_{m, n}
$$

A straightforward check [or use of eq. (31)] shows that this is indeed an ideal iff $\hat{m}=0$.

Is this ideal really non-trivial? It turns out that, for $\hat{n} \neq 1$ (including the case of main interest to us: $\hat{n}=0$ ), it is not! To see this, consider the (non-singular) linear map defined by $T_{i} \mapsto T_{i}^{\prime} \equiv-T_{i+\hat{i}}$ (note that for $\hat{n}=1$ this is not well defined). Since $\hat{m}=0$, this map transforms $\mathcal{J}$ to $\mathcal{A}_{m-1, n}$

$$
\left[T_{i}^{\prime}, T_{j}^{\prime}\right]=-(\widehat{i-j}) T_{i+j+(\hat{i}+\hat{j})}=-(\widehat{i-j}) T_{i+j+(\widehat{i+j})}=(\widehat{i-j}) T_{i+j}^{\prime}
$$

[the second equality follows from the fact that for $(\widehat{i-j}) \neq 0,(\widehat{i+j})=\hat{i}+\hat{j}]$, therefore, this map is an automorphism of Lie algebras, which means that $\mathcal{J}=\operatorname{sp}\left\{T_{m-2}\right\} \oplus \mathcal{A}_{m, n}$ is automorphic to $\mathcal{A}_{m-1, n}$.

\section{Construction Methods of Self-Dual Lie Algebras}

Our next goal is to show how the self-dual algebras described above (i.e., with $\hat{n}=0$ ) fit into the general family of self-dual algebras, and to clarify their significance. As noted in the introduction, it has been proved [0] that any self-dual Lie algebra can be constructed, using semi-simple and Abelian algebras, by a sequence of construction steps, each of which 
is either an orthogonal direct sum (i.e., a direct sum equipped with the natural direct sum metric) or a procedure called double extension [7]. This result seems, at first sight, to make all self-dual algebras available to us, but practically, this is not so, as we now explain.

The double extension of a self-dual Lie algebra $\mathcal{A}$ by another Lie algebra $\mathcal{B}$ (not necessarily self-dual) can be seen as a two-step process (we will give here only the information that will be needed later; more details can be found, for example, in [10]). The first step is to combine $\mathcal{A}$ and $\mathcal{B}$ to a semi-direct sum

$$
\mathcal{S}=\mathcal{B} \oplus \mathcal{A}
$$

(i.e., $\mathcal{S}$ is a direct sum of the vector spaces $\mathcal{B}$ and $\mathcal{A}, \mathcal{B}$ is a subalgebra, and $\mathcal{A}$ is an ideal) in such a way that the metric in $\mathcal{A}$ will be invariant also under the action of $\mathcal{B}$. The second step is the extension of $\mathcal{S}$ by an Abelian algebra $\mathcal{B}^{*}$ with $\operatorname{dim} \mathcal{B}^{*}=\operatorname{dim} \mathcal{B}$. The resulting algebra $\mathcal{D}$ has a Lie product of the following general form:

\begin{tabular}{c|ccc}
{$[\cdot, \cdot]$} & $\mathcal{B}$ & $\mathcal{A}$ & $\mathcal{B}^{*}$ \\
\hline $\mathcal{B}$ & $\mathcal{B}$ & $\mathcal{A}$ & $\mathcal{B}^{*}$ \\
$\mathcal{A}$ & $\mathcal{A}$ & $\mathcal{A}+\mathcal{B}^{*}$ & 0 \\
$\mathcal{B}^{*}$ & $\mathcal{B}^{*}$ & 0 & 0
\end{tabular}

For the first step one needs, in addition to the algebras $\mathcal{A}$ and $\mathcal{B}$, a representation of $\mathcal{B}$ as derivations in $\mathcal{A}$. Moreover, it was shown in [5] that if $\mathcal{B}$ acts through inner derivations (i.e., the action of each $y \in \mathcal{B}$ coincides with the adjoint action of an element $\hat{y} \in \mathcal{A}$ : $y: x \rightarrow[\hat{y}, x]$ ), the resulting algebra $\mathcal{D}$ is decomposable (i.e., expressible as an orthogonal direct sum). This means that for the construction of an indecomposable double extension, one needs knowledge about the outer (non-inner) derivations in $\mathcal{A}$, and such information is not available in general. Therefore a discovery of unknown (indecomposable) self-dual algebras is indeed significant.

Another method for constructing new self-dual Lie algebras is by performing a Wigner contraction [8] (this was proposed, in the context of string theory, in [9]). The initial data for this construction consists of a self-dual Lie algebra $\mathcal{S}_{0}$ and a subalgebra $\mathcal{B}_{0}$ of $\mathcal{S}_{0}$ such that the restriction of the metric on $\mathcal{S}_{0}$ to $\mathcal{B}_{0}$ is non-degenerate. Unlike in double extensions, the initial data needed here is very simple and generally available (for example, one can take $\mathcal{S}_{0}$ and $\mathcal{B}_{0}$ to be simple), therefore, the method can be easily used to find many new non-trivial self-dual algebras. It turns out, however [5], that the resulting algebra is always a double extension of an Abelian algebra. Actually, when $\mathcal{A}$ (in the process of double extension) is Abelian, the problem of the initial data described above does not exist [12] and one can indeed construct large families of double extensions of such algebras. Therefore the non-trivial task is to find (indecomposable, self-dual) deeper algebras (as they were called in [5]): algebras that their construction out of simple and one-dimensional algebras involves more than one double extension [13]. In the next section we show that almost all the algebras defined in section $\mathbb{1}$ are such algebras. 


\title{
V $\quad \mathcal{A}_{n}$ as a Deeper algebra
}

In the previous section we described the following inclusion relations among the indecomposable self-dual algebras:

\author{
\{ Indecomposable, Self-Dual Algebras \} \\ $\cup$ \\ \{(Single) Double-Extensions of Abelian Algebras \} \\ $\cup$ \\ \{ Algebras obtainable by a Wigner contraction $\}$.
}

The results of this section will imply that these are strict inclusions, i.e., all the three sets are distinct. Explicitly we will show here that among the algebras $\mathcal{A}_{n}$ with $\hat{n}=0$ (which

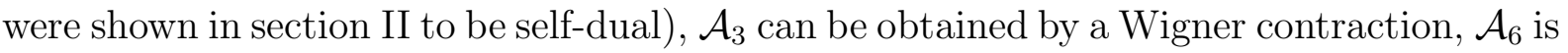
a double extension of an Abelian algebra but cannot be obtained by a Wigner contraction, and the rest are deeper algebras, i.e. they are not double extensions of Abelian algebras and, therefore, in particular, they cannot be obtained by a Wigner contraction.

First, from the list of the ideals found in section III we observe that $\mathcal{A}_{n}$ is indeed indecomposable [14]. Next, we try to identify in $\mathcal{A}_{n}$ the structure of a double extension of an Abelian algebra. The Lie product in an algebra $\mathcal{D}$ with such a structure is of the form

\begin{tabular}{c|ccc}
{$[\cdot, \cdot]$} & $\mathcal{B}$ & $\mathcal{A}$ & $\mathcal{B}^{*}$ \\
\hline $\mathcal{B}$ & $\mathcal{B}$ & $\mathcal{A}$ & $\mathcal{B}^{*}$ \\
$\mathcal{A}$ & $\mathcal{A}$ & $\mathcal{B}^{*}$ & 0 \\
$\mathcal{B}^{*}$ & $\mathcal{B}^{*}$ & 0 & 0
\end{tabular}

In this table we recognize two properties of $\mathcal{D}$ :

1. $\mathcal{D}$ is a semi-direct sum of $\mathcal{B}$ and the ideal $\mathcal{J}=\mathcal{A}+\mathcal{B}^{*}: \mathcal{D}=\mathcal{B} \in \mathcal{J}$;

2. $[\mathcal{J}, \mathcal{J}] \subset \mathcal{B}^{*}$, therefore, $\operatorname{dim}[\mathcal{J}, \mathcal{J}] \leq \operatorname{dim} \mathcal{B}^{*}=\operatorname{dim} \mathcal{B}$.

Consider the first property. The candidates for the ideal $\mathcal{J}$ were found in the previous section. It was shown that $\mathcal{J}=\mathcal{A}_{m, n}$ (possibly after an automorphic change of basis $\left.\left\{T_{i}\right\}\right)$. Following the same approach, we choose a basis $\left\{R_{i}\right\}_{i=0}^{m-1}$ for $\mathcal{B}$ such that $i$ is the minimal charge of $R_{i}$. $\left[T_{m-1}, T_{m-2}\right]=T_{2 m-3}$ and $2 m-3<n\left(\right.$ since $\left.\operatorname{dim} \mathcal{A}_{n} \geq 2 \operatorname{dim} \mathcal{B}\right)$, therefore, $\left[R_{m-1}, R_{m-2}\right] \neq 0$ and its minimal charge is $2 m-3$. $\mathcal{B}$ is closed under the Lie bracket and $\mathcal{B} \cap \mathcal{J}=\{0\}$, therefore, $\left[R_{m-1}, R_{m-2}\right] \notin \mathcal{J}$, which implies that $2 m-3<m$. This leaves us with $m=1$ or 2 [15].

As for the second property, we have

$$
\operatorname{dim}[\mathcal{J}, \mathcal{J}] \leq \operatorname{dim} \mathcal{B}=m
$$

One can easily verify that

$$
\left[\mathcal{A}_{m, n}, \mathcal{A}_{m, n}\right]=\mathcal{A}_{2 m+1, n}
$$


therefore, eq. (42) implies $n \leq 3 m$. On the other hand, $n+1 \geq 2 m$ (since $\operatorname{dim} \mathcal{A}_{n} \geq$ $2 \operatorname{dim} \mathcal{B})$. Recalling that $\hat{n}=0$, We obtain three possibilities:

$$
(m, n)=(1,3),(2,3),(2,6),
$$

and a direct check confirms that each of them indeed corresponds to a double extension of an Abelian algebra $\mathcal{A}$ (in the second possibility $\mathcal{A}$ is zero-dimensional). Observe that there is more than one way to represent an algebra as a double extension. Moreover, $\mathcal{A}_{6}$ can be obtained both by extending an Abelian algebra (with $m=2$ ) and by extending a non-Abelian algebra (with $m=1$ ), so the number of double extensions leading to a given Lie algebra is not unique [16].

Turning to the search of the structure of a Wigner contraction, the only candidates are those enumerated in (44). $\mathcal{A}_{3}$ is the Heisenberg algebra, and it is indeed a Wigner contraction of $s o(2,1) \oplus s o(2)$ [which leads to the first possibility in (44)]. The other candidate is $\mathcal{A}_{6}$, which corresponds to the last possibility in (44). To examine this case, we use the further requirement that in a Wigner contraction, $\mathcal{B}$ must be self-dual [17]. For $m=2, \mathcal{B}$ is the two-dimensional, non-Abelian Lie algebra,

$$
\left[R_{0}, R_{1}\right]=R_{1}
$$

This algebra is not self-dual, therefore, even if $\mathcal{A}_{6}$ can be obtained by a Wigner contraction, this procedure will not lead to an invariant metric on $\mathcal{A}_{6}$.

\section{Generalizations of the algebras $\mathcal{A}_{n}$}

We conclude with some comments about possible generalizations of the algebras defined in section [I], obtained by using the defining relations (5) with a different choice of the map "^". If one takes "^" to be some homomorphism from $\mathbb{Z}$ to some commutative ring $\mathbb{F}$ with unity, (6-8) hold, as well as (11) and one obtains a Lie algebra over $\mathbb{F}$. For example, one can take $\mathbb{F}=\mathbb{Z}_{p}$ ( $p$ a positive integer) with "^" being the natural homomorphism [18]. Another example is obtained by taking $\mathbb{F}=\mathbb{Z}$ and "^" the identity map, the result being the Virasoro algebra (with zero central charge). A different approach would be to keep $\mathbb{F}=\mathbb{Z}$ and to look for a map "^" (not necessarily a homomorphism) with the required properties. A natural candidate would be $\hat{i}=i \bmod p$ ( $p$ a positive integer). Taking $p=2$ and ${ }^{\wedge}: \mathbb{Z} \rightarrow\{0,1\}$, an analysis similar to the $p=3$ case leads to the choice $(i, j, k)=(1,0,0)$, for which the right-hand side of (11) does not vanish $\left(\hat{c}_{i j k}=\hat{c}_{k i j}=1\right.$,

$\left.\hat{c}_{j k i}=0\right)$. There seems to be no other choice of $p$ and range of the map "^" such that the multiplication is preserved.

In the previous sections we referred to the specific choice $\hat{i}=i \bmod 3 \in\{-1,0,1\}$, but actually section $\llbracket$ can be easily extended to a general commutative ring $\mathbb{F}$ with unity that has no zero-divisors and a general map "^" satisfying properties (6-8) and the Jacobi identity (11). The result is that the statement of the lemma is true whenever $\hat{3}=0$, while for $\hat{3} \neq 0$ (and, in particular, for $\hat{i}=i$ ) there is no invariant metric with only one diagonal [for $\hat{2}=0$, (21) fails while for $\hat{2}, \hat{3} \neq 0$, (23) fails]. In most of section [II the only additional 
assumption used is $\hat{3}=0$. Only the automorphism described at the end of the section assumes the explicit form of "^".

\section{Acknowledgments}

We would like to thank A. Reznikov and J. Bernstein for helpful discussions.

\section{References}

[1] M. A. Virasoro, Subsidiary conditions and ghosts in dual resonance models, Phys. Rev. D1 (1970) 2933.

[2] See, for example, V. G. Kač, Infinite-dimensional Lie algebras, 3rd ed. (Cambridge University Press 1993).

[3] V. G. Kač, Funct. Appl. 1 (1967) 328;

R. V. Moody, Bull. Am. Math. Soc. 73 (1967) 217.

[4] N. Mohammedi, On bosonic and super-symmetric current algebras for non-semisimple Lie groups, hep-th/9312182, Phys. Lett. B325 (1994) 371.

[5] J. M. Figueroa-O'Farrill and S. Stanciu, Non-semi-simple Sugawara constructions, hep-th/9402035, Phys. Lett. B327 (1994) 40.

[6] E. Witten, Non-Abelian bosonisation in two dimensions, Commun. Math. Phys. 92 (1984) 455 .

[7] A. Medina and Ph. Revoy, Algèbres de Lie et produit scalaire invariant, Ann. Sci. École Norm. Suppl. 18 (1985) 553.

[8] E. Inonu and E. P. Wigner, On the contraction of groups and their representations, Proc. Natl. Acad. Sci. USA 39 (1953) 510.

[9] D. I. Olive, E. Rabinovici and A. Schwimmer, A class of string backgrounds as a semi-classical limit of WZW models, hep-th/9311081, Phys. Lett. B321 (1994) 361.

[10] These algebras were used, for the construction of field-theoretical models, in

A. Giveon, O. Pelc and E. Rabinovici, WZNW models and gauged WZNW models based on a family of solvable Lie algebras, hep-th/9509013, Nucl. Phys. B462 (1996) 53.

[11] The $\Longleftarrow$ direction of (8) follows from (7), but for the other direction (7) only implies

$$
(\hat{i-j})=0 \Longrightarrow \hat{i}-\hat{j}=0 \bmod 3
$$


and the stronger result $\hat{i}-\hat{j}=0$ follows from the fact that $|\hat{i}-\hat{j}|$ is always at most 2 . When $(\hat{i-j}) \neq 0$ this reasoning breaks down and indeed we have, e.g., $\hat{2}-\hat{1} \neq(\widehat{2-1})$.

[12] For Abelian $\mathcal{A}$, any (non-trivial) linear map in $\mathcal{A}$ is obviously an outer derivation, and to construct a double extension one only needs to worry about the invariance of the metric (the second step of the construction is completely determined by the first one and poses no further difficulties).

[13] One might also consider a double extension of a reductive algebra $\mathcal{A}$, i.e., an orthogonal direct sum of Abelian and semi-simple algebras. However, as shown in [5], the semi-simple factor of $\mathcal{A}$ factorizes also in the result $\mathcal{D}$ (because a semi-simple algebra does not have outer derivations), therefore, the result in this case is decomposable.

[14] This means [7] that they should be expressible as double extensions by the onedimensional algebra, and this structure can be indeed easily identified with:

$$
\mathcal{B}=\operatorname{sp}\left\{T_{0}\right\} \quad, \quad \mathcal{B}^{*}=\operatorname{sp}\left\{T_{n}\right\} \quad, \quad \mathcal{A}=\mathcal{A}_{1, n} / \mathcal{B}^{*} .
$$

[15] The value $m=0$ is also a possibility, but it is not interesting. It corresponds to $\operatorname{dim} \mathcal{B}=0$. As a double extension it means not to do anything - remaining with the (Abelian) algebra $\mathcal{A}$ one started with. As a Wigner contraction it means that, starting with some self-dual Lie algebra $\mathcal{S}_{0}$, all we did is to set its Lie bracket to 0 , so that we end up with the Abelian Lie algebra of the same dimension, which is trivially self-dual. In the present context, this corresponds to the one-dimensional algebra: $\mathcal{D}=\mathcal{A}_{0}$.

[16] The notion of "depth" of a self-dual Lie algebra, suggested in [5], is still well defined, if one allows only extensions by either a simple- or a one-dimensional algebra. Alternatively, the depth can be defined as the minimal number of double extensions.

[17] More precisely, in a Wigner contraction (unlike double extension), the metric is not involved at all in the construction of the algebra itself. However, the metric of the initial algebra $S_{0}$ is the source of the metric of the resulting algebra, and it turns out (see, for example, the presentation in $[10]$ ) that if we want the final metric to be non-degenerate, $\mathcal{B}$ must be self-dual.

[18] This example, however, is irrelevant for string-theoretical models, since there one needs an algebra over $\mathbb{R}$ and for this $\mathbb{F}$ must be some sub-ring of $\mathbb{R}$. 